\title{
Design of Safety Monitoring and Early Warning System for Buried Pipeline Crossing Fault
}

\author{
Wu Liu ${ }^{1, a}$, Wanggang Hou ${ }^{1, b^{*}}$, Wentao Zhang ${ }^{1, c}$ \\ ${ }^{1}$ Petroleum \& Natural Gas Engineering Institute, \\ Southwest Petroleum University, Chengdou 610500, China

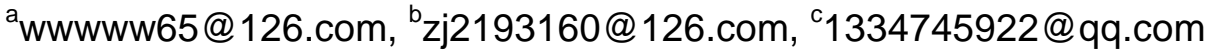

\begin{abstract}
Keywords: fault; buried pipelines; monitor; early warning; system; safety
Abstract. Long-distance buried pipeline crossing fault is prone to being cracked, fractured and buckling failure, so the implementation of pipeline security monitoring and early warning can not only after the earthquake quick report, but provide basis for assessing the safe operation condition of the pipeline and the emergency rescue of risk tube section. To monitor fault hazards body, the pipeline bearing body and the force of soil-pipe through the advanced sensor, forming the function of ground acceleration early warning, fault displacement early warning, pipeline strain early warning, pipeline leakage warning, location of pipeline inspection, real time display of the deformation and stress of pipe, the dynamic changes of the relative displacement and the interaction force of the pipe-soil. It can effectively reduce the pipe damage caused by the sudden disasters and the occurrence of secondary damage.
\end{abstract}

\section{Introduction}

With the comprehensive construction of long-distance oil and gas pipelines, the pipelines are inevitably affected by various geological disasters, and earthquake experiences have shown that the greatest impact on buried pipelines is caused by surface fault rupture [1].

At present, the domestic and foreign scholars have carried on the theory and the experiment research deeply on the behavior of the buried pipeline under the action of fault, then putting forward corresponding seismic measures, but the long-term and dynamic monitoring of the pipeline crossing fault is still less [2].Therefore, to carry out pipeline safety monitoring and early warning to improve the safety level of the pipeline and avoid the secondary disasters caused by the leakage of oil and gas pipeline is of great significance.

\section{Application status of domestic and international monitoring system}

Foreign oil and gas pipeline seismic monitoring system is mainly used in the Alaska pipeline, gas pipeline network in Tokyo, Russia's Sakhalin 2 pipeline and Caspian, eastern Turkey, Iran pipeline [3]. Alaska earthquake monitoring system consists of 11 digital seismic acceleration recorders which monitor ground motion intensity mainly and determine the safety of the pipeline [4]. The seismic monitoring system of the Tokyo gas network is composed of a sensing automatic isolation system and a pipeline earthquake prediction system [5]. Domestic earthquake monitoring and early-warning system has been used less on oil and gas pipeline, so far, only Ji Ning segment of the West-East natural gas pipeline [6] and China Myanmar pipeline during the early stage of the construction were installed monitoring system.

The seismic monitoring system is developed to monitor the relative displacement on both sides of the fault and the pipeline strain from the initial only monitoring intensity of ground motion. However, monitoring system is more concerned about the monitoring of seismic fault zone, and the selection of the location of the pipeline strain monitoring is not reasonable enough; cannot real-time, accurately display the deformation of any point along the pipeline; the lack of monitoring of soil and pipeline interaction; cannot leak positioning when the pipe leaks. Therefore, it is necessary to study the seismic fault and pipeline monitoring and early warning system. 


\section{Design of pipeline monitoring and early warning system}

The fault hazards body, pipeline bearing body and the force of soil-pipe respectively are to be monitored by monitoring and early warning system, and evaluating the security status of the pipeline comprehensively, accurately and rapidly. The system is composed of 4 parts, which are the sensor subsystem, data acquisition and transmission subsystem, data analysis and processing subsystem, and early warning subsystem. The peak acceleration of ground motion, the displacement of the fault, the strain of the pipeline, the displacement of the pipeline, the temperature of the pipeline and the force of the soil are monitored, collecting field data and transmitting the date to the remote control center through wireless 4G and Beidou satellite dual-channel, and processing and disseminating early warning information at the monitoring center.Figure. 1 is a safety monitoring and early system for pipeline crossing fault.

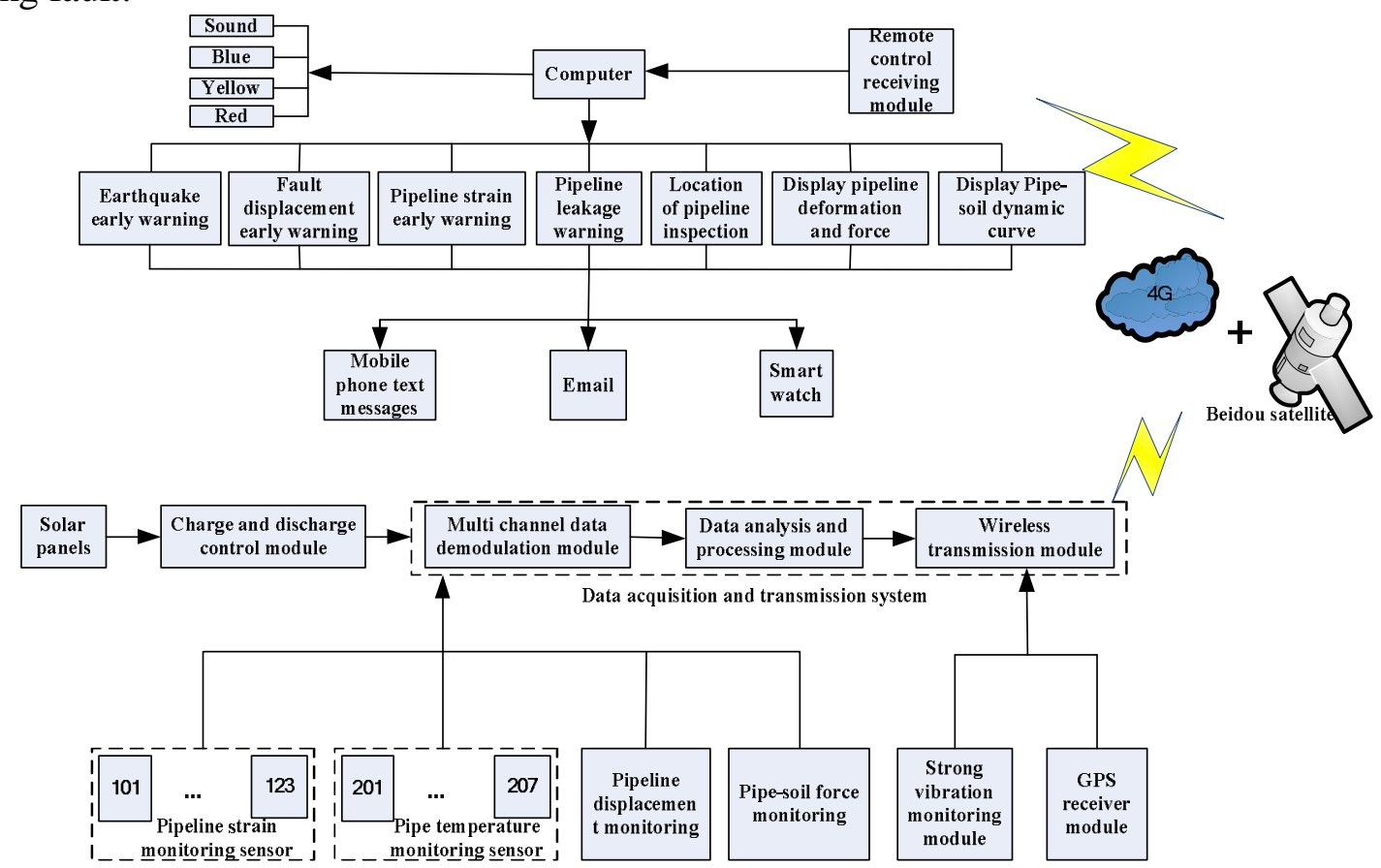

Fig.1 Structural diagram of the monitoring and early warning system for pipeline crossing fault

\section{Monitoring sensor subsystem}

Monitoring sensor system consists of strong vibration monitoring unit, GPS fault displacement monitoring unit, pipeline strain monitoring unit, pipeline displacement monitoring unit, the pipeline operating temperature monitoring unit and pipe-soil interaction monitoring unit.

\section{( 1 ) Strong vibration monitoring unit}

Digital strong motion instruments are installed in the pumping station, the valve chamber or strong motion stations near the fault zone $(20 \mathrm{~km}$ from the fault rupture surface) to monitor peak ground acceleration. When peak ground acceleration is equal to or greater than $0.2 \mathrm{~g}$ but less than $0.3 \mathrm{~g}$, for primary warning; when the peak acceleration is equal to or greater than $0.3 \mathrm{~g}$, for secondary warning, based on "Seismic technical code for oil and gas transmission pipeline engineering"[7].

\section{( 2 ) Pipeline strain monitoring unit}

The stress and strain of the pipeline can reflect the influence of the earthquake on the safety of the pipeline. Because stress analyzer which is used to monitor the stress of the pipeline is necessary to excavate the pipeline, remove the pipeline coating, and the cost high [8], the strain monitoring of pipeline is carried out by using the FBG strain gauge.

The allowable tensile(allowable strain when the pipe is yielding) and ultimate tensile strain(strain at failure) of the pipeline are determined by theoretical calculation or experimental study. The pipeline is in a safe state when the strain monitored is less than the allowable tensile strain; when the strain 
monitored is equal to or greater than the allowable tensile strain but is less than the ultimate tensile strain, the pipeline is in a state of insecurity, and primary warning is carried out; when the strain monitored reaches the limit strain, the pipeline is in failure state, for secondary warning.

2) Determining the location of strain gage installation along the pipeline

Calculating stress and strain concentration point of the pipeline and installing strain gauge based on the field surveyed data. Figure 2 is the selection of strain monitoring points for pipeline under the action of strike slip fault.

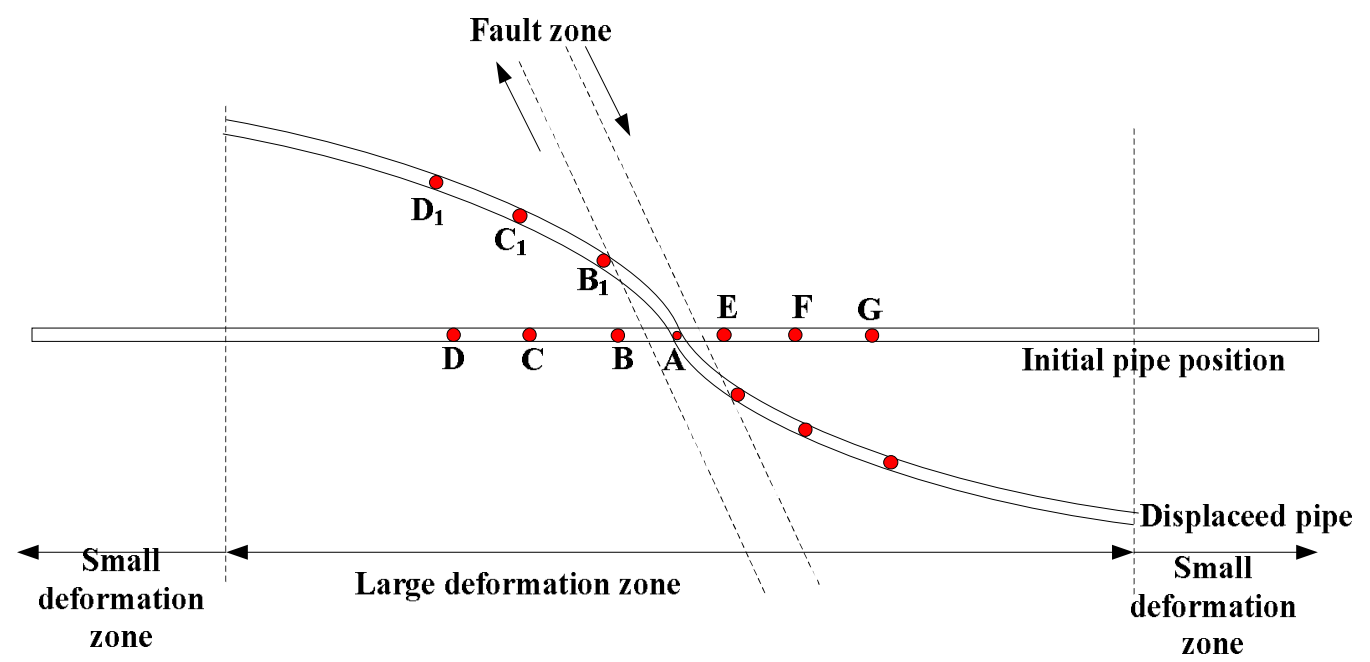

Fig.2 Location of strain monitoring points of pipeline crossing strike slip fault

The figure above, A, B, C, D is the pipeline strain monitoring point at one side of fault, $B_{1}, C_{1}, D_{1}$ is pipeline deformed position $\mathrm{B}, \mathrm{C}, \mathrm{D}$ after fault movement. After fault movement, point $\mathrm{A}$ is the axial tensile stress-strain maximum and point $\mathrm{C}_{1}$ is the maximum point of the total stress of the pipeline (including the axial stress and the bending stress), So selecting point $\mathrm{A}$ and $\mathrm{C}$ for monitoring. In order to avoid the change of fault distance which leads to the total stress position in the axial direction changing, adding $\mathrm{B}_{1}$ and $\mathrm{D}_{1}$ to be monitored. Therefore, $\mathrm{A}, \mathrm{B}, \mathrm{C}, \mathrm{D}$ are pipeline strain monitoring points of the fault side, for the soil on both sides of the same strike slip fault, the pipeline deformation is anti-symmetric, E, F, G the same choice.

( 3 ) GPS fault displacement monitoring unit

Two high precision GPS receivers are installed on the two plates of the fault, and the relative displacement of the fault is monitored. The relationship between fault displacement and pipeline strain is studied by numerical simulation, then establishing fault displacement and pipeline strain database. By constantly changing the relative displacement of the two plates, $\mathrm{H}_{1}, \mathrm{H}_{2}, \mathrm{~V}_{1}, \mathrm{~V}_{2}$ are determined to be thresholds for the horizontal direction of the fault and the vertical direction of the fault distance. When the horizontal direction of fault is $\mathrm{H}_{1}$ or vertical to the fault distance $\mathrm{V}_{1}$, the pipe can reach the allowable tensile strain, for primary warning; When the fault level reaches $\mathrm{H}_{2}$ or $\mathrm{V}_{2}$, for secondary warning.

( 4 ) Pipeline operating temperature monitoring unit

Powerful seismic waves often lead to pressure wave of leakage difficult to be observed when an earthquake occurs. So the fiber Bragg grating temperature sensor is installed in the stress and strain concentration point of the pipeline to locate leakage point according to the leakage of fluid thermal radiation when there is a leak in the pipeline.

( 5 ) Monitoring unit of relative displacement and interaction force of pipe-soil

The monitoring date of the relative displacement and the interaction force can be used to quantitatively evaluate the influence of the pipeline on the soil movement, and to provide basic data for the development of the mechanical model of the pipe-soil interaction. 


\section{Data acquisition and transmission system}

The automatic collection and effective transmission of the data is the key of pipeline safety monitoring. With the development of communication industry, the latest $4 \mathrm{G}$ communication speed and communication flexibility, and is a good wireless transmission channel, but it is too dependent on communication base station to prone to traffic congestion or paralysis. Considering that China's Beidou satellite has been achieved in the whole territory of seamless coverage, high data transmission security without ground disaster and environmental restrictions etc. advantages. The dual channel communication with the combination of $4 \mathrm{G}$ and Beidou satellite is proposed.

\section{Data processing and management system}

Data processing and management is the core of the whole system. Four early warnings are carried out to prevent the failure or delay of a single early warning function; at the same time, the dynamic display of the deformation and the stress state of the pipeline is carried out; the pipe-soil forces and relative displacement variation data is monitored to provide the basis for theoretical research.

\section{Early warning system}

Carrying out sound and light alarm and releasing early warning information through mobile phone, e-mail or smart watches, when the fault hazards body or the pipeline bearing body reaches the threshold value. Among them, the first level warning for sound and blue flash; the two - level warning is a sound and a yellow flash; the leakage warning is a sound and a red flash.

\section{Summary}

The design of buried pipeline monitoring and early warning system provides a new way for the traditional pipeline seismic method, which can not only reduce the pipeline damage in the earthquake, but also can quickly determine the safe operation of the pipeline, and release the early warning information.

The fault hazard body, pipeline, and the interaction force between pipe-soil are monitored dynamically by sensors and data acquisition equipment to evaluate pipeline safety. Providing basic data for the theoretical analysis of the reaction behavior of the pipeline and conducting multi-level early warning. Producing sound and light alarm and using SMS, e-mail or smart Watch to issue warning information when the pipeline is in a state of insecurity, to provide the best repair time for the staff.

\section{References}

[1] Wang Bin, Study on analytical methods of buried steel pipelines under active faults, Dalian University of Technology, Dalian, 2011.

[2] J Zh Huang, Y H Yang, Liu Wei, et al, A safety monitoring and early-warning system for buried pipelines crossing seismic fault belts, Natural gas industry, 33(2013) 151-157.

[3] J P Liu, L W Fu, J B Hao, et al, Application and technology of earthquake monitoring and early warning for long-distance oil and gas pipeline, World earthquake engineering, 26(2010) 176-181.

[4] Nyman, D. J., E. L. Nelson, C. H. Roach, New trans-Alaska pipeline earthquake monitoring system, Optimizing Post-Earthquake Lifeline System Reliability, ASCE, 1999.

[5] Shimizu, Yoshihisa, et al, Development of real-time safety control system for urban gas supply network, Journal of geotechnical and geoenvironmental engineering, 132(2006) 237-249.

[6] Zh Y Li, F Sh Huang, Y X Xu, Introduction to seismic design of the fracture zone of the Ji ing pipeline project, Exchange of science and technology, 10(2006) 69-71. 
[7] GB50470-2008 Seismic technical code for oil and gas transmission pipeline engineering, 2009.

[8] China Petroleum Pipeline Company, Risk management technology of oil and gas pipeline geological hazard, Petroleum Industry Press, Beijing, 2010. 